\title{
The Role of Theory in Construction Management: A Call for Debate.
}

\author{
David Seymour, Darryl Crook and John Rooke,
}

\author{
Published as: \\ Seymour, D., Crook, D. \& Rooke, J. (1997) 'The Role of Theory in Construction \\ Management: A Call for Debate,' Construction Management and Economics, 15(1):117-119.
}

In this short paper we intend to express certain questions about the theoretical basis of construction management as an academic discipline and, by implication, its relationship to construction management practice. It is hoped in this way to stimulate a debate in these pages which will contribute both to the academic status of the discipline and to the usefulness of its contributions to practice. It is our feeling that such a debate is long overdue and that as the leading journal in the field, CM\&E provides the logical arena in which to conduct it.

In a recent study of the first ten years of the journal, Betts and Lansley (1993) outline the development of the discipline. They conclude that one of the characteristics of a mature discipline is a body of objective knowledge in the form of a theoretical base, and suggests that 'when a subject begins to experience research into its research [...] this signifies that an underlying theory about the discipline is starting to evolve' (op cit p.222).

Betts and Lansley are less than satisfied with the extent to which this has taken place in the pages of $\mathrm{CM} \& \mathrm{E}$, suggesting that 'the discipline is becoming rather inward-looking, selfreferential and lacking in its guidance from and contribution to theory' (ibid). This, they acknowledge, may be compensated for by the empirical nature of the research which is being carried out. They find that 'seventy percent of the papers [studied] are based on original or nearly original data gathered through case studies or as truly empirical data' (op cit p.241).

In classifying the contribution to theory of published works in construction management, their study adopted a five point scale, starting at 'Insights. The contribution lies largely in the data [...] presented-the papers do not generate new models or theories'. The second level is 'Model testing or fitting. The testing of statistical or organizational models', moving to 'Model building. Developing complex (largely static) new models [...] for forecasting and decision making'. The next level is 'System building. Developing complex (largely dynamic or interactive) systems [...] for operations management and decision making'. Finally there is 'Theory building/modifying. Development or modification of theory, for example whilst application of mainstream management theory to construction would fall in [sic] model testing category, developments of that theory to fit construction would fall here' (op cit p.245).

Two interesting points arise from this classification: first, that there is a hierarchy of contributions to the discipline, and second, that 'theory' within construction management is only produced at one level of this hierarchy. It is this level of theory building/modifying that they suggest has been inadequately addressed by the construction management community. 
We welcome these calls to develop the theoretical base of the discipline and agree that this issue must be addressed to a greater degree than it currently is. However, we see a prior need to enquire as to what the nature of this theoretical base should be. If construction management is to develop into a rigorous academic discipline, the nature of its theoretical base, and the methods used to arrive at its theory, are crucial issues. As an empirical science, 'designed to yield verifiable knowledge of human group life and human conduct' (Blumer 1969 p.21), construction management must meet a fundamental criterion. This is well expressed by Blumer:
'an empirical science presupposes the existence of an empirical world. Such an empirical world exists as something available for observation, study, and analysis. It stands over against the scientific observer, with a character that has to be dug out through observation, study and analysis. This empirical world must forever be the central point of concern. It is the point of departure and the point of return in the case of empirical science. It is the testing ground for any assertions made about the empirical world. "Reality" for empirical science only exists in the empirical world, can be sought only there, and can be verified only there [...]' (op cit p.21, italics in original)

In other words, the nature of construction management practice, that is what construction management 'is', what it means to 'do' construction management, must be the starting point, the focus, the culmination and the arbitor of all theoretical activity.

We have previously suggested that construction management is dominated by research carried out under a 'rationalist paradigm'. This paradigm has important implications for the type of theory seen as important in construction management, and also the methods of investigation used by researchers in this field. Thus, it is assumed that the reality of management practice can be captured in the form of a single objective account, taking the form of a generic causal representation of the 'system' (Seymour and Rooke 1995). It appears to be something of this nature which Betts an Wood-Harper (1994) have in mind when they quote Hammer and Champy's definition of a business process as "a collection of activities that takes one or more kinds of input and creates an output that is of value to the customer." This kind of theory building is facilitated by various forms of survey research, which typically produce statistical data relating correlations between variables.

Our suggested alternative is to concentrate upon the interpretative methods which researchers and managers use to make sense of the world. This approach yields an investigation which is primarily concerned with meaning, rather than causality and produces an account which recognises the respective viewpoints of practitioners in the process. It is our belief that such an account better reflects the realities of construction management as a practice. It is our perception that investigation into the nature of construction management, as a practice, has not taken place. Construction management, as a discipline, has simply accepted without significant question one particular meaning of 'theory,' and one particular method for arriving at that theory.

In the two works cited above (Betts and Lansley, 1993; Betts and Wood-Harper, 1994) we see a distinction being drawn between empirical research, which does not contribute to theory and 'scientific' research, which does. In the absence of any discussion of the nature of the theory being advocated, we assume that, in line with the conceptions of the rationalistic paradigm, it is intended to be similar to that found in the natural sciences: global, objective and verifiable. If this is not the case, then the onus rests with the authors to explain what theory should consist of. 
According to Schutz (1971), the method of scientific theorising involves the suspension, or bracketing, of the theorist's awareness of his or her personal situation. This enables the achievement of an objective, depersonalised knowledge. This anonimity of the researcher is guaranteed by the creation of a theoretical framework, every term of which is empirically accountable and in conformity with the tenets of formal logic. In order that anonymity is maintained, the theorist must eschew the use of unexamined common sense concepts in his or her explanations.

We suggest that construction management is not amenable to such an approach. The discipline of construction management is crucially different from that of a natural science, in that the our 'objects of study' are people. As such they attribute meaning both to what they do, and to the presence of the researcher. In the natural sciences, the objects of study do not attribute meaning, allowing the community of theorists to impose its own meanings upon phenomena. In management research, meaning is intersubjectively created and managed between researcher and researched, in an iterative interpretive process. In such a process, the identity and style of the researcher are integral to the production of data. Furthermore, this process is impossible without the use of the common sense concepts and methods which we all use and take for granted in our everyday lives.

The difficulty is compounded since the discipline intends to produce advice for practitioners. This requires theorists to make value judgements, a process logically incompatable with the attempt to acheive a depersonalised objectivity. We contend that insufficient attention has been paid to the seperation of these conflicting aims.

In our view, research in construction management has tended to underestimate, or ignore the importance of the interpretive process. Thus, the all-important analysis which goes into the identification of problems, the formulating of questions, the catagorisation of informants and respondents, the attributing of significance to actions and answers, are all glossed over and hidden. The effect of this is that the 'data,' which are often treated with explicit mathmatical analyses, have already been subjected to a sophisticated and unexamined process of preparation, before the reported research commences.

The interpretive methods which researchers in construction management use to investigate the world are no different from those used by managers, or for that matter, from the methods of ordinary members of society. Indeed, since the vast majority of our data consists of what managers tell us, the relationship between the manager and researcher is best characterised as one between an instructor and instructee. The only advantage we can reasonably claim as academics, is that we have leisure to think more closely about our methods and our findings than the managers we research. The results of such activity simply do not represent theories in the scientific sense.

This is not to say that construction management cannot support theoretical work, or that it cannot become a rigorous academic discipline, with accepted and objective means of 'finding out.' Research which acknowledges its interpretive context has an important contribution to make in this regard. However, in order that this be achieved, we must reconsider our conceptions of 'theory', 'rigour', 'objectivity', etc. What is needed we would argue, is not so much the creation of new and better causal models, but a logical analysis of the concepts which have been used to build these models.

In conclusion, while welcoming recent attempts to assess the theoretical maturity of the discipline, we have questioned certain pre-theoretical assumptions upon which these attempts are based. Our intention, as stated at the outset, has been to provoke a response. If we 
endorse Betts and Lansley's assertion that the existence of a theoretical base signifies the maturity of a discipline, we would add that a further indicator of such maturity is the presence of scholarly debate in its leading journals. 


\section{References}

BETTS, M. \& LANSLEY, P., (1993), 'Construction Management and Economics: A review of the first ten years’, in Construction Management and Economics, 11:221-245.

BETTS, M. \& WOOD-HARPER, T., (1994), 'Re-engineering construction: a new management research agenda’, in Construction Management and Economics, 12:551-556.

BLUMER, H., (1969), Symbolic Interactionism-Perspective and Method, Prentice Hall International, London.

CME, (1996), ‘Aims and Scope’, in Construction Management and Economics, 14:1.

SCHUTZ, A., (1971), 'Common Sense and Scientific Interpretation of Human Action,' in Collected Papers 1, Martinus Nijhoff, The Hague.

SEYMOUR, D.E., \& ROOKE, J.A., (1995), 'The culture of the industry and culture of research', in Construction Management and Economics, 13:511-523. 